8. Soukas, A., Cohen, P., Socci, N.D., and Friedman, J.M. 2000. Leptin-specific patterns of gene expression in white adipose tissue. Genes Dev. 14:963-980

9. Way, J.M., et al. 2001. Comprehensive messenger ribonucleic acid profiling reveals that peroxisome proliferator-activated receptor gamma activation has coordinate effects on gene expression in multiple insulin-sensitive tissues. Endocrinology. 142:1269-1277.

10. Xu, H., et al. 2003. Chronic inflammation in fat plays a crucial role in the development of obesity-related insulin resistance. J. Clin. Invest.
112:1821-1830. doi:10.1172/JCI200319451.

11. Weisberg, S.P., et al. 2003. Obesity is associated with macrophage accumulation in adipose tissue. J. Clin. Invest. 112:1796-1808. doi:10.1172/ JCI200319246.

12. Yuan, M., et al. 2001. Reversal of obesity- and diet-induced insulin resistance with salicylates or targeted disruption of Ikkbeta. Science. 293:1673-1677.

13. Uysal, K.T., Wiesbrock, S.M., Marino, M.W., and Hotamisligil, G.S. 1997. Protection from obesityinduced insulin resistance in mice lacking TNFalpha function. Nature. 389:610-614.
14. Perreault, M., and Marette, A. 2001. Targeted dis ruption of inducible nitric oxide synthase protects against obesity-linked insulin resistance in muscle. Nat. Med. 7:1138-1143.

15. Maeda, N., et al. 2002. Diet-induced insulin resistance in mice lacking adiponectin/ACRP30. Nat. Med. 8:731-737

16. Tong, Q., et al. 2000. Function of GATA transcription factors in preadipocyte-adipocyte transition. Science. 290:134-138.

17. Sierra-Honigmann, M.R., et al. 1998. Biological action of leptin as an angiogenic factor. Science 281:1683-1686.

\title{
A radical explanation for glucose-induced $\beta$ cell dysfunction
}

\section{Michael Brownlee}

Diabetes Research Center, Albert Einstein College of Medicine, New York, New York, USA

The development of type 2 diabetes requires impaired $\beta$ cell function. Hyperglycemia itself causes further decreases in glucose-stimulated insulin secretion. A new study demonstrates that hyperglycemia-induced mitochondrial superoxide production activates uncoupling protein 2 , which decreases the ATP/ADP ratio and thus reduces the insulin-secretory response (see the related article beginning on page 1831). These data suggest that pharmacologic inhibition of mitochondrial superoxide overproduction in $\beta$ cells exposed to hyperglycemia could prevent a positive feed-forward loop of glucotoxicity that drives impaired glucose tolerance toward frank type 2 diabetes.

J. Clin. Invest. 112:1788-1790 (2003). doi:10.1172/JCI200320501.

\section{The diabetes epidemic and its consequences}

Diabetes and impaired glucose tolerance currently affect an estimated 29 million people in the US (1). For those born in 2000, the estimated lifetime risk of developing diabetes is 36\% (2). People with diabetes have large reductions in life expectancy and in quality of life (2), due to diabetes-specific microvascular complications in the retina, renal glomerulus, and peripheral nerve, and to extensive atherothrombotic macrovascular disease affecting arteries that supply the heart, brain, and lower extremities. It has

Address correspondence to: Michael Brownlee, Diabetes Research Center, Albert Einstein College of Medicine, 1300 Morris Park Avenue, New York, New York 10461, USA. Phone: (718) 430-3636;

Fax: (718) 430-8570;

E-mail: Brownlee@aecom.yu.edu.

Conflict of interest: The author has declared that no conflict of interest exists.

Nonstandard abbreviations used: tricarboxylic acid (TCA); reduced flavin adenine dinucleotide $\left(\mathrm{FADH}_{2}\right)$; uncoupling protein (UCP). been estimated that up to $70 \%$ of patients with acute myocardial infarction have either diabetes or impaired glucose tolerance (3).

As a consequence of its microvascular pathology, diabetes is the leading cause of blindness, end-stage renal disease, and a variety of debilitating neuropathies. Diabetics are the fastest-growing group of renal dialysis and transplant recipients, and in the US, their 5-year survival rate is only 21 percent, worse overall than that for all forms of cancer combined. Over $60 \%$ of diabetic patients suffer from neuropathy, which accounts for $50 \%$ of all nontraumatic amputations in the US (4).

\section{Insulin resistance, $\beta$ cell function, and the natural history of type 2 diabetes}

Both genetic and environmental factors (mainly obesity) contribute to insulin resistance. Recent work with tissue-conditional knockouts of both Glut4 and the insulin receptor in mice have shown that adipose tissue plays a central role in the pathogenesis of insulin resistance, and that there is significant crosstalk among insulin target tissues (5).

Insulin resistance induces a compensatory increase in $\beta$ cell mass, which in many people results in normal glucose levels. In other people, intrinsic defects in this compensatory $\beta$ cell response prevent adequate compensation, and impaired glucose tolerance or type 2 diabetes occurs. Impaired glucose tolerance leads to type 2 diabetes in a significant number of people, and type 2 diabetes, in turn, becomes progressively unresponsive to oral antidiabetic agents, until treatment with insulin is necessary.

\section{Glucose toxicity and the decline of $\beta$ cell function}

Hyperglycemia is widely recognized as the causal link between diabetes and diabetic complications (6). More recently, adverse effects of hyperglycemia on insulin target tissues and on pancreatic $\beta$ cells have also been recognized, and this phenomenon has been termed "glucotoxicity." Chronic hyperglycemia has been shown to induce multiple defects in $\beta$ cells, including early decreases in glucose-stimulated insulin secretion, and late irreversible changes in insulin-gene transcription and $\beta$ cell mass $(7,8)$. In patients with impaired glucose tolerance, lowering of glucose levels dramatically reduces the progression to type 2 diabetes (9), suggesting that glucotoxicity plays a major role in this transition.

The central role of mitochondria in glucose-stimulated insulin secretion Pancreatic $\beta$ cells sense the ambient plasma glucose concentration because (a) the high- $K_{m}$ glucose transporter GLUT2 facilitates rapid equilibration across the cell membrane, and (b) the 


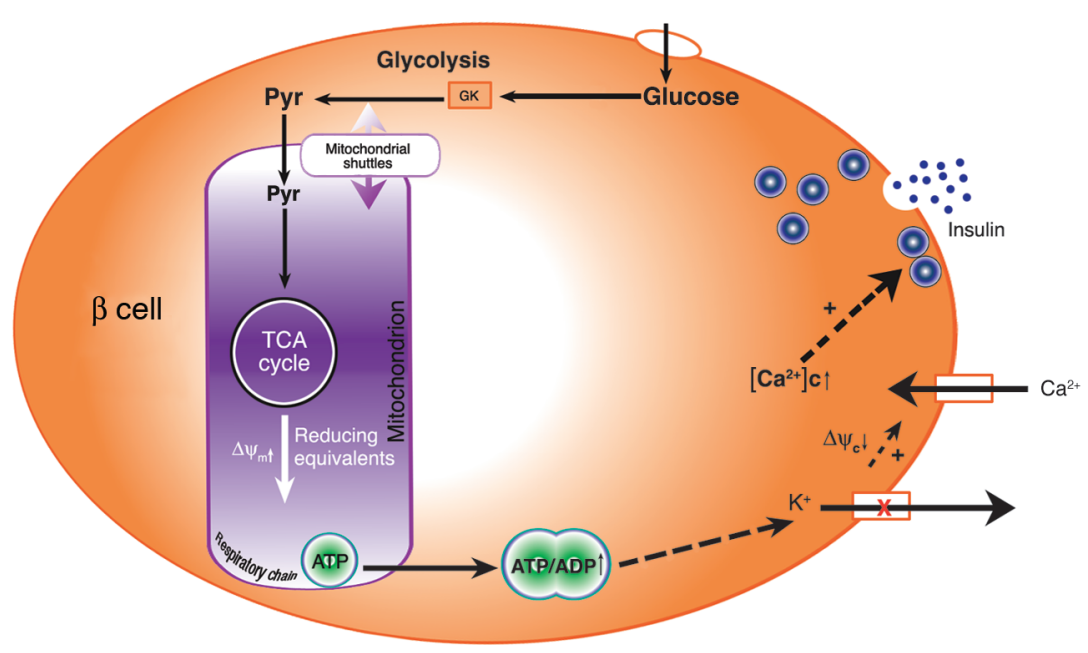

high- $K_{m}$ hexokinase isoform glucokinase allows the generation of a proportionate signal through glycolytic and mitochondrial metabolism of glucose (10). This results in an increased ATP/ADP ratio, which closes an ATPsensitive potassium channel in the cell membrane, thereby depolarizing the cell membrane and activating a voltage-gated calcium channel. The resultant influx of calcium triggers secretion of insulin granules (Figure 1).

The glucose-sensitive increase in the ATP/ADP ratio is caused by greater electron flux through the mitochondrial electron-transport system (Figure 2). Pyruvate derived from glycolysis is transported into the mitochondria, where it is oxidized by the tricarboxylic acid (TCA) cycle to produce NADH and reduced

\section{Figure 2}

Effect of hyperglycemia on mitochondrial electron-transport chain function in the pancreatic $\beta$ cell. Hyperglycemia increases production of electron donors from the tricarboxylic acid (TCA) cycle (NADH and FADH ${ }_{2}$ ). This increases the membrane potential $\left(\Delta \mu_{H^{+}}\right)$, because protons are pumped across the mitochondrial inner membrane in proportion to electron flux through the electrontransport chain. Inhibition of electron transport at Complex III by increased $\Delta \mu_{\mathrm{H}^{+}}$ increases the half-life of free radical intermediates of coenzyme Q, which reduce $\mathrm{O}_{2}$ to superoxide. Krauss and colleagues (13) have demonstrated that hyperglycemia-induced mitochondrial superoxide activates UCP2mediated proton leak, thus lowering ATP levels and impairing glucose-stimulated insulin secretion. Figure modified with permission from Nature (15). $\mathrm{P}_{\mathrm{i}}$, inorganic phosphorus. flavin adenine dinucleotide $\left(\mathrm{FADH}_{2}\right)$. Mitochondrial NADH and $\mathrm{FADH}_{2}$ provide energy for ATP production via oxidative phosphorylation by the electron-transport chain.

Electron flow through the mitochondrial electron-transport chain is carried out by four inner membraneassociated enzyme complexes, plus cytochrome $c$ and the mobile carrier coenzyme Q. NADH derived from the TCA cycle donates electrons to Complex I. Complex I ultimately transfers its electrons to coenzyme Q. Coenzyme $\mathrm{Q}$ is also reduced by electrons donated from several $\mathrm{FADH}_{2}$-containing dehydrogenases, such as the TCA cycle succinate:ubiquinone oxidoreductase (Complex II). Electrons from reduced coenzyme $\mathrm{Q}$ are then transferred to Complex III. Electron trans-

\section{Figure 1}

Model of glucose-stimulated insulin secretion in the pancreatic $\beta$ cell. Following phosphorylation by glucokinase (GK), glucose is converted to pyruvate by glycolysis. Pyruvate enters the mitochondria and fuels the TCA cycle, resulting in the transfer of reducing equivalents to the respiratory chain, hyperpolarization of the mitochondrial membrane, and ATP generation. Subsequent closure of KATP channels depolarizes the cell membrane, which opens voltage-gated calcium channels, increasing the concentration of cytosolic calcium $([\mathrm{Ca} 2+] \mathrm{c})$. This influx of calcium triggers insulin release from the cell. Figure modified with permission from Nature (17). Pyr, pyruvate; $\Delta \psi \mathrm{m}$, mitochondrial membrane potential; $\Delta \psi c$, cell membrane potential.

port then proceeds through cytochrome $c$, Complex IV, and, finally, molecular oxygen.

Electron transfer through Complexes I, III, and IV generates a proton (voltage) gradient. Much of the energy of this voltage gradient $\left(\Delta \mu_{\mathrm{H}}^{+}\right)$is used to generate ATP, as the collapse of the proton gradient through ATP synthase (Complex V) drives the ATP synthetic machinery. This energy can also be dissipated as heat through the mediation of uncoupling proteins (UCPs). When the electrochemical potential difference generated by this proton gradient is high, electron transport in Complex III is partially inhibited, resulting in a backup of electrons to coenzyme Q and their donation to molecular oxygen, leading to increased generation of the free radical superoxide.

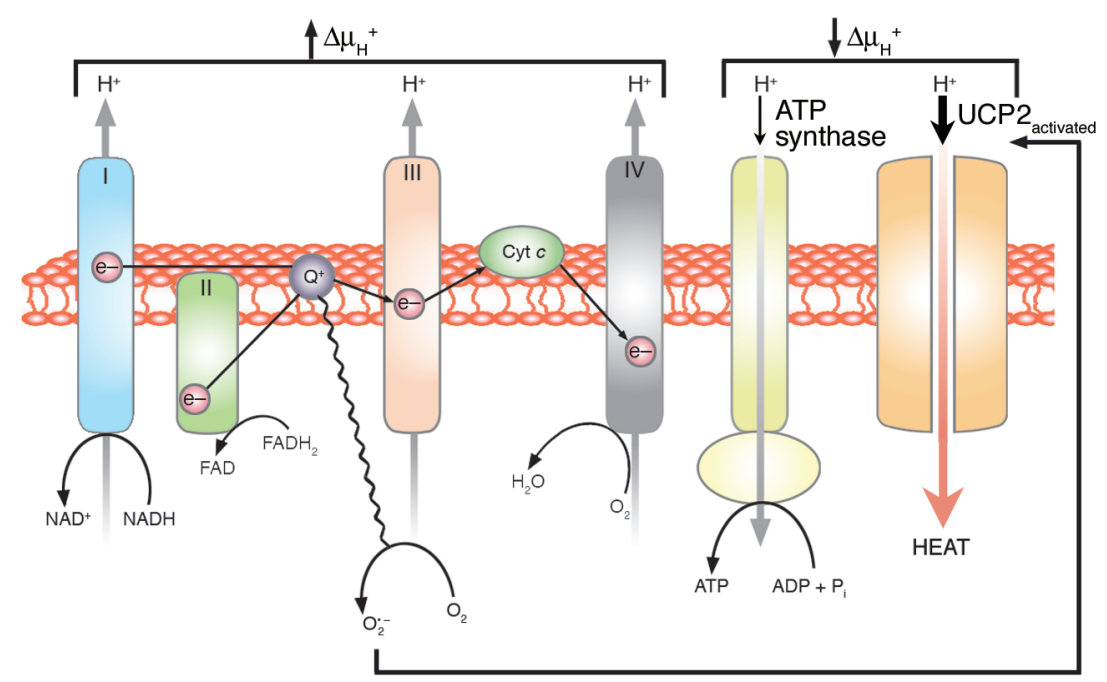


Excess activation of UCP2 by superoxide causes $\beta$ cell dysfunction How does hyperglycemia cause $\beta$ cell dysfunction? The answer to this question has not been clear. Although elegant studies by Zhang and coworkers (11) have suggested that the level of UCP2 gene expression in $\beta$ cells is an important determinant, conflicting data in the literature suggested that changes in expression alone are not the whole story (12).

In this issue of the JCI, Krauss et al. (13) now show that the key missing element in hyperglycemia-induced $\beta$ cell dysfunction is activation of UCP2. This UCP2 activation is accomplished by hyperglycemia-induced superoxide formation by the mitochondrial electron-transport chain. In vitro studies have suggested that superoxide could activate UCPs (14), but these data have been somewhat controversial. Now, Krauss et al. demonstrate that under physiologic conditions, endogenous superoxide generated by hyperglycemia activates UCP2. This activation diverts energy away from ATP synthesis (Figure 2), thereby decreasing the ATP/ADP ratio. This results in impaired glucose-stimulated insulin secretion (13).

\section{Therapeutic implications and future directions}

The data reported by Krauss et al. (13) have enormous clinical implications. They suggest that pharmacologic inhibition of mitochondrial superoxide overproduction in $\beta$ cells exposed to hyperglycemia could prevent the positive feed-forward loop of glucotoxicity that pushes impaired glucose tolerance into frank type 2 diabetes. Since the incidence and rate of progression of diabetic complications increase in proportion to the level of hyperglycemia (6), prevention or even significant delay of the transition from impaired glucose tolerance to type 2 diabetes would have a major positive impact on diabetes-associated morbidity and mortality. Interestingly, the process of hyperglycemia-induced overproduction of superoxide by the mitochondrial electron transport chain also appears to be the central mechanism underlying all the major molecular mechanisms implicated in glucosemediated vascular damage $(15,16)$. Thus, a common unifying mechanism may underlie hyperglycemic damage in $\beta$ cells, endothelial cells, and other targets of glucotoxicity.

An important aspect of $\beta$ cell glucose toxicity that remains to be clarified is the possible role of hyperglycemiainduced superoxide production by the mitochondria and subsequent activation of UCP 2 in long-term loss of $\beta$ cell mass. Reactive oxygen leads to irreversible decreases in the level of the transcription factor PDX-1 (8), which is critical for insulin-gene expression, and also for $\beta$ cell neogenesis.

Future studies on the role of altered mitochondrial production of both superoxide and ATP, based on the work of Krauss et al. (13), may also lead to a better understanding of the mechanisms underlying the inexorable decline of $\beta$ cell insulin production and $\beta$ cell mass with increased duration of type 2 diabetes.

\section{Acknowledgments}

Michael Brownlee is supported by grants from the NIH, the American Diabetes Association, and the Juvenile Diabetes Research Foundation.
1. Cowie, C.C., et al. 2003. Prevalence of diabetes and impaired fasting glucose in adults: United States, 1999-2000. JAMA. 290:1702-1703.

2. Narayan, K.M., et al. 2003. Lifetime risk for diabetes mellitus in the United States. JAMA 290:1884-1890.

3. Norhammar, A., et al. 2002. Glucose metabolism in patients with acute myocardial infarction and no previous diagnosis of diabetes mellitus: a prospective study. Lancet. 360:2140-2144.

4. National Diabetes Data Group. 1995. Diabetes in America. 2nd edition. Bethesda, Maryland, USA. NIH Publication no. 95-1468. 1-730.

5. Minokoshi, Y., et al. 2003. Tissue-specific ablation of the GLUT4 glucose transporter or the insulin receptor challenges assumptions about insulin action and glucose homeostasis. J. Biol. Chem. 278:33609-33612.

6. 1993. The effect of intensive treatment of diabetes on the development and progression of long-term complications in insulin-dependent diabetes mellitus. The Diabetes Control and Complications Trial Research Group. N. Engl. J. Med. 329:977-986.

7. Gerich, J.E., and Smith, T.S. 2003. $\beta$-Cell defects and pancreatic abnormalities in type 2 diabetes In Textbook of diabetes. 3 rd edition. J.C. Pickup and G. Williams, editors. Blackwell Science. Oxford, United Kingdom. 23.1-23.11

8. Robertson, R.P., et al. 2003. Glucose toxicity in $\beta$-cells: type 2 diabetes, good radicals gone bad, and the glutathione connection. Diabetes. 52:581-587.

9. Knowler, W.C., et al. 2002. Reduction in the incidence of type 2 diabetes with lifestyle intervention or metformin. N. Engl. J. Med. 346:393-403.

10. Maechler, P., and Woheim, C.B. 2001. Mitochondrial function in normal and diabetic $\beta$-cells. Nature. 414:807-812.

11. Zhang, C.-Y., et al. 2001. Uncoupling protein-2 negatively regulates insulin secretion and is a major link between obesity, $\beta$-cell dysfunction and type 2 diabetes. Cell. 105:745-755.

12. Polonsky, K.S., and Semenkovich, C.F. 2001. The pancreatic $\beta$-cell heats up: UCP2 and insulin secretion in diabetes. Cell. 105:705-707.

13. Krauss, S., et al. 2003. Superoxide-mediated activation of uncoupling protein 2 causes pancreatic $\beta$ cell dysfunction. J. Clin. Invest. 112:1831-1842. doi:10.1172/JCI200319774.

14. Echtay, K.S., et al. 2002. Superoxide activates mitochondrial uncoupling proteins. Nature. 415:96-99.

15. Brownlee, M. 2001. Biochemistry and molecular cell biology of diabetic complications. Nature. 414:813-820.

16. Du, X., et al. 2003. Inhibition of GAPDH activity by poly(ADP-ribose) polymerase activates three major pathways of hyperglycemic damage in endothelial cells. J. Clin. Invest. 112:1049-1057. doi:10.1172/JCI200318127.

17. Maechler, P., and Wollheim, C.B. 2001. Mitochondrial function in normal and diabetic $\beta$-cells. Nature. 414:807-812. 